\title{
Use of Ultrananocrystalline Diamond as a Phase-contrast Aperture Material
}

Robert M Glaeser ${ }^{1}$, David Larson ${ }^{2}$, Benjamin Muddiman ${ }^{3}$, Simone Sassolini ${ }^{3}$, Stefano Cabrini ${ }^{3}$, and Jian $\mathrm{Jin}^{4}$

${ }^{1 .}$ Life Sciences Division, LBNL, University of California, Berkeley, Ca

2. JCAP, LBNL, University of California, Berkeley, Ca

3. The Molecular Foundry, LBNL, University of California, Berkeley, Ca

4. Engineering Department, LBNL, University of California, Berkeley, Ca

Boron-doped "ultrananocrystalline diamond" (UNCD), available as a $2 \mu \mathrm{m}$-thick layer on a "diamond on insulator" wafer (Advanced Diamond Technologies, Romeoville, IL), is investigated as a potential material for making devices that can be placed in the back focal plane of the objective lens of a TEM, the goal being to produce high contrast for in-focus images of weak phase objects. The resistance of diamond to knock-on damage when irradiated, even at $300 \mathrm{keV}$, is an attractive feature. In addition, diamond (indeed, carbon in general) is not expected to form a native oxide that is thicker than a monolayer. The ultrananocrystalline form of diamond is favored over a microcrystalline form because unwanted, "patchy" variations in surface potential (due to differences in the work-function for different crystal facets) are expected to decay more rapidly, i.e. on a length scale comparable to the domain size. We believe, however, that care must be taken to compensate for the contact potential (Galvani potential) between the UNCD device layer and the supporting (boron-doped) silicon wafer (handle).

The tulip aperture [1] used here was made by first back-etching a window in the silicon with $\mathrm{KOH}$ followed by $\mathrm{HF}$ etch to remove the $\mathrm{SiO}_{2}$ "stopping layer", thus leaving a self-supported membrane of UNCD. A hard mask was then created on the top surface of the window by electron-beam lithography, after which unwanted UNCD was removed by oxygen plasma etch. Finally, the hard mask was removed with a second HF etch, leaving the bare, self-supporting tulip aperture shown in Figure 1.

In preliminary results obtained so far, we found that the edge of a UNCD aperture exhibits significant charging when it is intentionally irradiated by a focused beam of $300 \mathrm{keV}$ electrons at a temperature $\sim 300{ }^{\circ} \mathrm{C}$ (Figure 2), but no such charging occurs when the same experiment is done at a temperature $>600{ }^{\circ} \mathrm{C}$ (data not shown). In addition, after heating the aperture $>600{ }^{\circ} \mathrm{C}$, the edge no longer exhibited charging when it was again irradiated at $\sim 300{ }^{\circ} \mathrm{C}$ (data not shown). This behavior suggests that (1) irradiation of UNCD at lower temperatures produces a Volta potential due to local changes in the chemical and structural composition of the surface, as has been suggested to happen for amorphous carbon [2], and (2) heating to $>600{ }^{\circ} \mathrm{C}$ produces a global change in the surface, which makes the aperture immunne to further changes when irradiated. Still to be determined is whether applying a bias voltage to the UNCD "membrane", relative to the silicon frame (handle) will compensate the charging shown in Figure 3, currently thought to be due to the contact potential mentioned above [3].

\section{References:}

[1] B Buijsse et al, Ultramicroscopy 111 (2011), p. 1688.

[2] R Danev et al, PNAS 111 (2014), p. 15635.

[3] This work was supported in part by NIH grant GM083039 and by User Project \#3016 at The Molecular Foundry, LBNL. 


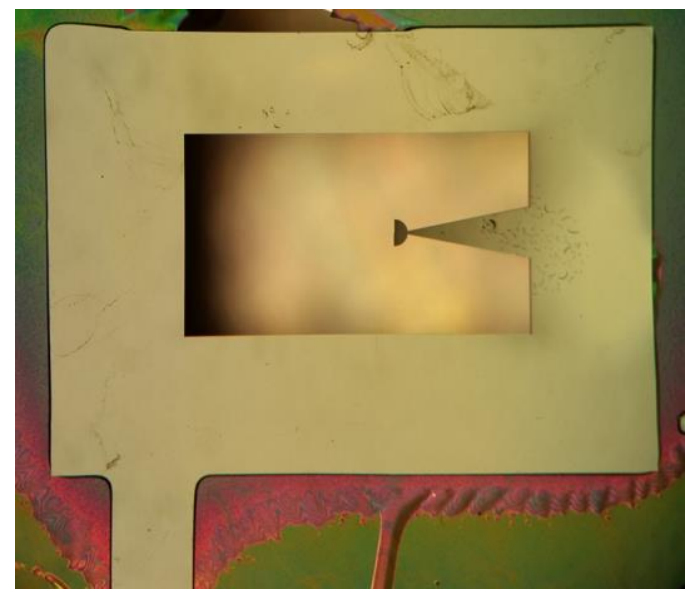

Figure 1. Optical image of our first UNCD "tulip" aperture. The dimensions of the rectangular, open aperture (with the tulip-like feature protruding in from the right) are $\sim 0.5 \mathrm{~mm}$ by $\sim 0.8 \mathrm{~mm}$. The smooth, UNCD layer is closest to the viewer, supported on a silicon frame (handle), which is visible around the periphery. A $1 \mu \mathrm{m}$-thick $\mathrm{SiO}_{2}$ layer remains sandwiched between the UNCD layer and the silicon in the area outside the window that was etched into the silicon.
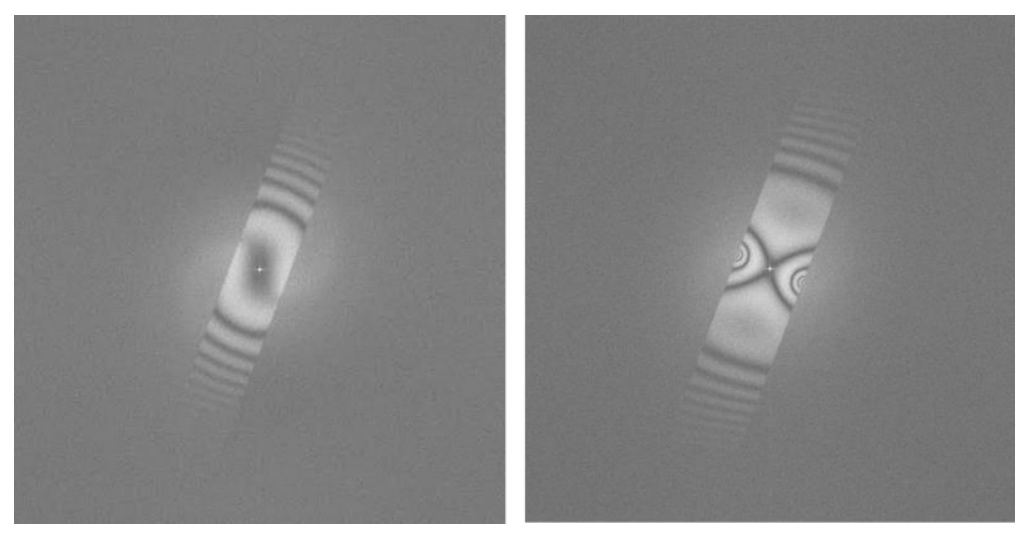

Figure 2. Power spectra of images of a thin, amorphous carbon film, recorded with the tulip aperture at a temperature of $\sim 300{ }^{\circ} \mathrm{C}$. Intentionally touching an edge of the aperture with the focused, unscattered electron beam creates a local charging effect, manifested by a strong distortion (RIGHT) of the usual, focusdependent phase-contrast transfer function (LEFT).
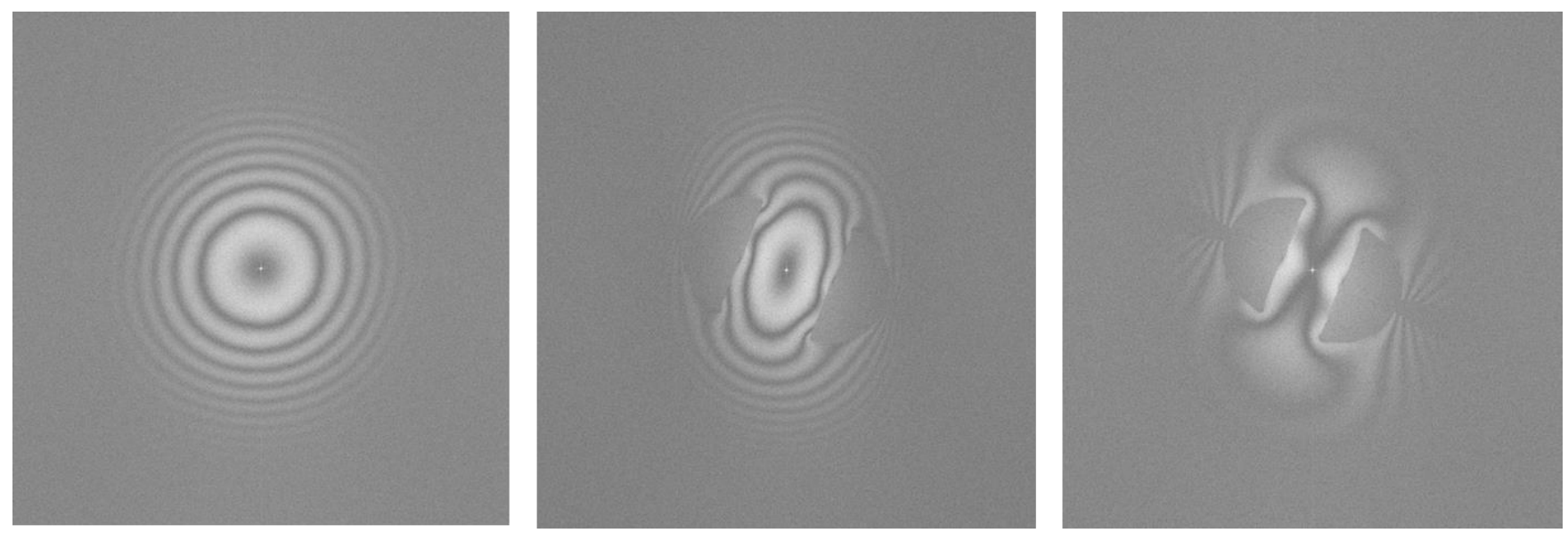

Figure 3. Power spectra of images of a thin, amorphous-carbon specimen. While the phase-contrast effect is well described by the usual defocus term when the electron diffraction pattern is in the open area of the aperture (LEFT), significant phase distortions occur close to the single-sideband (tulip-like) feature. Images were recorded at the previous defocus value (CENTER) and closer to focus (RIGHT). 